\title{
A Sliding Mode Observer design for the Average State Estimation in Large-Scale Systems
}

\author{
Alessandro Pilloni, Diego Deplano, Alessandro Giua, Elio Usai
}

\begin{abstract}
In this paper the average state estimation problem on a large-scale system with only few gateway nodes available for measurements is solved as the design problem of a reducedorder sliding mode observer subjected to unmatched perturbations. Necessary and sufficient conditions to guarantee the exact estimation are derived. Still, even in the case such conditions are not fulfilled, the boundedness of the estimation error is proved. Finally, a criteria to minimize such bound, and a gain adaptation law to trade-off that bound and its convergence rate are provided. Neither the observer complexity nor the estimation error depend explicitly on the size of the system, thus making the approach scalable and computationally tractable. Simulations supporting the effectiveness of the proposed strategy and a comparison with other existing strategies are also provided.

Index Terms-Average estimation, large-scale systems, slid-
\end{abstract} ing mode observers, functional observers, state estimation.

\section{INTRODUCTION}

The state estimation is a critical task on Large-Scale Systems (LSSs) since it requires a dense deployment of computational resources and many sensing gateway devices [1]. Thus, to reduce the infrastructure's costs while maintaining computationally tractable the complexity of the estimators, since decades, either decentralized [2], or clustered-oriented reduced-order observers are under developments [3], [4].

Moreover, in many monitoring applications the full state information is neither necessary nor possible due to potential observably deficiencies. Thus, the estimation of some aggregated quantities of the state is often sufficient. Here we focus on the estimation of the average state of an LSS, which is of interest in different field, such as the monitoring of the mean traffic density in some portion of a communication or a road network to prevent its congestion [5], the mean proportion of infected people during the spreading of an epidemic [6], the mean circulation of leaders opinions over geographic areas [7], the mean temperature in smart buildings through a sensor network [8] to mention a few.

Literature review The problem of estimating the average quantity of the states of an LSS has been outlined for first in [9], as special case of [10]. Therein, it is proposed an

Manuscript received March 4, 2021; revised May 7, 2021; accepted May 21,2021 . The research leading to these results has received funding by the Sardinian Regional Government, from project "Virtual Energy" under call "Cluster top-down actions (POR FESR)", and Project RASSR05871 MOSIMA, FSC 2014-2020, annuity 2017, Subject area 3, Action Line 3.1., and POR SARDEGNA FSE 2014-2020-Asse III, Azione 10.5.12, "Avviso di chiamata per il finanziamento di Progetti di ricerca Anno 2017".

All the authors are with the Department of Electrical and Electronic Engineering, University of Cagliari, Italy.

E-mail: \{alessandro.pilloni,diego.deplano,giua,elio.usai\}@unica.it. observer reduction approach for the redesign of Luenbergerlike functional observers [11], by means of suitably chosen bi-orthogonal projection matrices. Projection matrices not only allows to reduce the model dimension to be considered, but the play also a key role in the design of general functional observers [12]. As a drawback, low-order projections introduce matched and unmatched unknown perturbations in the observers' error dynamics which need to be compensated. The use of projection strategies is also further motivated by the fact that LSSs are often not observable due to the limited number of measurements nodes. In [13] conditions for the average observability on an LSS are outlined. Then, in [14], reduced-order open-loop and the closed-loop linear average estimators are presented, and the needed operating conditions on an LSS to achieve the task are discussed. Complementary results consisting in the identification of clusters in LSSs enabling the average estimation [4], in the analysis of the subgraph induced by the unmeasured nodes in negative uniform networks [6], presence of outliers [15], as well as other functionals estimation [16] are further available.

Contrarily to the mentioned researches, which share the fact to consider only linear strategies, and/or Luenbergerlike observers, here we aim at solving the average state estimation by means of Sliding-Mode Observers (SMOs). Our choice comes from the consideration that SMOs are globally recognized as a privileged technique for the robust state estimation on uncertain processes affected by unknown inputs. This is because of their inherent robustness against matched [17], [18] and unmatched [19], [20] disturbances and uncertainties, their resilience to measurement noise similar to that offered by Kalman filters, and finally, contrarily to control applications, the chattering phenomena in SMOs is considered a minor problem. Moreover, by the analysis of the so-called equivalent injection signals useful estimations also on the unknown inputs introduced by the mentioned low-order projections can be obtained and thus exploited to improve the performance of standard average estimators.

Main contributions The paper's contribution is threefold: 1) the average state estimation on an LSS is solved as the design problem of a reduced-order SMO subjected to unknown inputs; 2) necessary and sufficient conditions for the exact average-state estimation despite the presence of both matched and unmatched unknown inputs introduced by the used low-order model projection are derived; 3) even when those conditions are not fulfilled, the estimation error boundedness is proved; then, a criteria to minimize such bound, along with a gain adaptation criteria to trade-off that 
bound and its convergence rate are derived.

Structure of the paper The paper's notation is introduced at the end of this section. The problem statement is outlined in Section II. In Section III the proposed SMO design is presented, and all the main results discussed in detail. Simulations over different test-cases are illustrated in Section IV. Finally, in Section V concluding remarks are outlined.

Notation The sets of natural, real and complex numbers are denoted by $\mathbb{N}, \mathbb{R}$ and $\mathbb{C}$. Let $n$ and $m \in \mathbb{N}$, then $\mathbf{0}_{n \times m}$, and $\mathbf{1}_{n \times m}$, are $n \times m$ matrices with all 0 , and 1 , entries. If $m=1$, it can be omitted and they reduce to column vectors, whereas $\boldsymbol{I}_{n}$ is the identity matrix of dimension $n$. Vector $\boldsymbol{x}=\left\{x_{i}\right\} \in \mathbb{R}^{n}$ is intended to be column vectors, and its entries are $x_{i} \in \mathbb{R}, i=1, \ldots, n$. The transpose of $\boldsymbol{x}$ is $\boldsymbol{x}^{\top}$. The absolute value is denoted by $|\cdot|$ and a $p$-norm for vectors by $\|\cdot\|_{p}$. Let $M \in \mathbb{R}^{n \times m}$ be a matrix with independent columns, its pseudo-inverse is $\boldsymbol{M}^{+}=\left(\boldsymbol{M}^{\top} \boldsymbol{M}\right)^{-1} \boldsymbol{M}^{\top}$. If $\boldsymbol{M}$ is square, $\operatorname{eig}(\boldsymbol{M})$ is its eigenvalue set, and $\operatorname{rank}(\boldsymbol{M})$ denotes its rank. The operator $\operatorname{sign}(\boldsymbol{x})$ is the set-valued signoperator which outputs a vector whose $i$-th entry is 1 if $x_{i}>0,-1$ if $x_{i}<0,[-1,1]$ otherwise.

\section{PROBLEM Formulation}

Consider an LSS composed of $n \in \mathbb{N}$ subsystems with commensurable states, assumed scalar for simplicity $x_{i} \in \mathbb{R}$. The pattern of interconnection is described by $\boldsymbol{A}=\left\{a_{i j}\right\} \in$ $\mathbb{R}^{n \times n}$, where $a_{i j}=0$ if the $i$-th node is not instantaneously influenced by the $j$-th node. The LSS is influenced by $m \in \mathbb{N}$ external inputs $u_{\ell} \in \mathbb{R}$ through $\boldsymbol{B}=\left\{b_{i \ell}\right\} \in \mathbb{R}^{n \times m}$. Thus, in accordance with [9], each node satisfies

$$
\dot{x}_{i}(t)=\sum_{j=1}^{n} a_{i j} x_{j}(t)+\sum_{\ell=1}^{m} b_{i \ell} u_{\ell}(t) .
$$

This general description encompasses several well-known LSSs models, such as reaction-diffusion systems [21], compartmental systems [22], multi-agent systems [23] to mention a few. Let us now consider only $p$ over the $n$ nodes equipped with higher-level sensing and communication facilities. Without loss of generality, the LSS state vector $\boldsymbol{x}=\left[x_{1}, \ldots, x_{n}\right]^{\top} \in \mathbb{R}^{n}$ can be partitioned as $\boldsymbol{x}=\left[\boldsymbol{x}_{u}^{\top}, \boldsymbol{y}^{\top}\right]^{\top}$, where $\boldsymbol{y} \in \mathbb{R}^{p}$ and $\boldsymbol{x}_{u} \in \mathbb{R}^{k}$ denotes the measured and unmeasured states, and $k=n-p$. The statespace representation of the resulting LSS is as follows

$$
\begin{aligned}
{\left[\begin{array}{c}
\dot{\boldsymbol{x}}_{u}(t) \\
\dot{\boldsymbol{y}}(t)
\end{array}\right] } & =\stackrel{\left.\begin{array}{cc}
\boldsymbol{A}_{11} & \boldsymbol{A}_{12} \\
\boldsymbol{A}_{21} & \boldsymbol{A}_{22}
\end{array}\right]}{\left[\begin{array}{c}
\boldsymbol{x}_{u}(t) \\
\boldsymbol{y}(t)
\end{array}\right]+\left[\begin{array}{c}
\boldsymbol{B}_{1} \\
\boldsymbol{B}_{2}
\end{array}\right]} \boldsymbol{u}(t) \\
\boldsymbol{y}(t) & =\underbrace{\left[\begin{array}{ll}
\mathbf{0}_{p \times k} & \boldsymbol{I}_{p}
\end{array}\right]\left[\begin{array}{c}
\boldsymbol{x}_{u}(t) \\
\boldsymbol{y}(t)
\end{array}\right]}_{\boldsymbol{C} \in \mathbb{R}^{p \times n}}
\end{aligned}
$$

where $\boldsymbol{u}=\left[u_{1}, \ldots, u_{m}\right]^{\top} \in \mathbb{R}^{m}$ stacks the system inputs, $\boldsymbol{y} \in \mathbb{R}^{p}$ the available measurements, and $\boldsymbol{A}_{i j}$ and $\boldsymbol{B}_{\ell}$ are matrices with proper dimensions, with $i, j, \ell=1,2$.

Problem 1 (Average state estimation) Consider the LSS in Eq. (2) whose states are partitioned into unmeasured $\boldsymbol{x}_{u} \in \mathbb{R}^{k}$ and measured $\boldsymbol{y} \in \mathbb{R}^{p}$. Our aim is to design an observer that, by means of the available measurements on $\boldsymbol{y} \in \mathbb{R}^{p}$, and $\boldsymbol{u} \in \mathbb{R}^{m}$, enables the estimation of the averaged profile of the unmeasured states, given by

$$
a(t)=\frac{1}{k} \mathbf{1}_{k}^{\top} \boldsymbol{x}_{u}(t) .
$$

In general, in an LSS only few nodes make their measurements available at a higher-level for monitoring purposes, namely $p \ll n$. In particular, the average estimation problem becomes more challenging in the case the LSS in Eq. (2) is neither observable nor detectable, because this in turn implies the estimation of Eq. (3) cannot be solved by means of standard full-state observers. It is also worth to remark such observability deficiency is guaranteed if $p<n / 2$ [13].

This prompts to seek functional observers, which enable by means of low-order projections the estimation of functions of the system state. This is sufficient in a large number of applications and leads to more computationally tractable observers [10]. Following the related literature [14]-[9], $a(t)$ in Eq. (3) is made explicit by means of the next projection

$$
\boldsymbol{z}(t)=\boldsymbol{P} \boldsymbol{x}(t), \text { where } \boldsymbol{P}=\left[\begin{array}{cc}
\frac{1}{k} \mathbf{1}_{k}^{\top} & \mathbf{0}_{p}^{\top} \\
\mathbf{0}_{p \times k} & \boldsymbol{I}_{p}
\end{array}\right] \in \mathbb{R}^{(p+1) \times n},
$$

and $\boldsymbol{z}=\left[a, \boldsymbol{y}^{\top}\right]^{\top}$. Then, from Eqs. (2) and (4) one derives

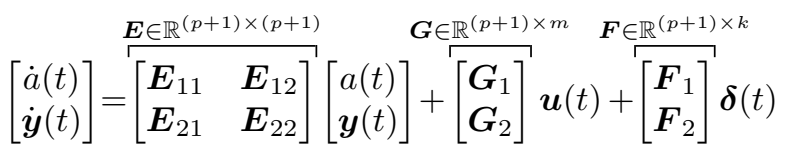

where $\boldsymbol{E}_{i j}, \boldsymbol{G}_{\ell}, \boldsymbol{F}_{\ell}$ with $i, j, \ell=1,2$ are matrices with proper dimension in accordance to the next partition

$$
\boldsymbol{E}=\left[\begin{array}{cc}
\frac{1}{k} \mathbf{1}_{k}^{\top} \boldsymbol{A}_{11} \mathbf{1}_{k} & \frac{1}{k} \mathbf{1}_{k}^{\top} \boldsymbol{A}_{12} \\
\boldsymbol{A}_{21} \mathbf{1}_{k} & \boldsymbol{A}_{22}
\end{array}\right], \boldsymbol{G}=\left[\begin{array}{c}
\frac{1}{k} \mathbf{1}_{k}^{\top} \boldsymbol{B}_{1} \\
\boldsymbol{B}_{2}
\end{array}\right], \boldsymbol{F}=\left[\begin{array}{c}
\frac{1}{k} \mathbf{1}_{k}^{\top} \boldsymbol{A}_{11} \\
\boldsymbol{A}_{21}
\end{array}\right] .
$$

and $\boldsymbol{\delta}(t)$ plays as an unmatched unknown input, such that

$$
\boldsymbol{\delta}(t)=\left(\boldsymbol{I}_{k}-\frac{1}{k} \mathbf{1}_{k} \cdot \mathbf{1}_{k}^{\top}\right) \boldsymbol{x}_{u}(t) \in \mathbb{R}^{k} .
$$

In particular, $\boldsymbol{F} \boldsymbol{\delta}(t)$ in Eq. (5) accounts for what is missed due to the projection of the vector $\boldsymbol{x} \in \mathbb{R}^{n}$ to the subspace $z \in \mathbb{R}^{p+1}$ and vice-versa. From Eq. (6), one may further note that $\boldsymbol{\delta}(t)$ represents the element-wise deviation of each entry of $\boldsymbol{x}_{u}(t)$ to the average profile $a(t)$. Thus, it yields that

$$
\mathbf{1}_{k}^{\top} \boldsymbol{\delta}(t)=0, \quad \forall t \in \mathbb{R} .
$$

\section{A. Working assumptions}

The need to estimate $a(t)$ in Eq. (5) leads to Assumption 1.

Assumption 1 The pair $\left(\boldsymbol{E}, \boldsymbol{C P}^{+}\right)$is observable.

Although the projection in Eq. (4) makes explicit $a(t)$ despite also potential observability deficiencies of the LSS in Eq. (2), $\boldsymbol{P} \in \mathbb{R}^{(p+1) \times n}$ is rectangular thus, differently from conventional state observation problems, $\nexists \boldsymbol{P}^{-1}$. Since the mapping of a vector in $\mathbb{R}^{n}$ to a subspace in $\mathbb{R}^{p+1}$ loss information, clearly that information cannot be also recovered by means of the pseudo-inverse projection $\boldsymbol{x}=\boldsymbol{P}^{+} \boldsymbol{z}$. That amount of missed information, enclosed in the term $\boldsymbol{F} \boldsymbol{\delta}(t)$ in Eq. (5), plays thus as a state-dependent unknown input vector. Follows that every unstable trend of $\boldsymbol{\delta}(t)$ (or 
equivalently of $\boldsymbol{x}_{u}(t)$ ) will destabilize the low-order dynamic in Eq. (5). Since $\boldsymbol{\delta}(t)$ cannot be recovered from $\boldsymbol{y}$, this makes also impossible the stabilization of any low-order estimation error dynamic through $\boldsymbol{y}$. This leads to Assumption 2, that is also required in the related works, cf. [13]-[14], [9], [10].

Assumption 2 The LSS in Eq. (2) is stable and the inputs are bounded, namely one of the following holds:

- $\operatorname{eig}(\boldsymbol{A}) \subset \mathbb{C}_{\leq 0}$ and $\int_{0}^{\infty}\|\boldsymbol{u}(t)\| d t<\infty$,

- $\operatorname{eig}(\boldsymbol{A}) \subset \mathbb{C}_{<0}$ and $\|\boldsymbol{u}(t)\|<\infty$ for all $t \in \mathbb{R}$

\section{MAIN RESUlT}

We propose now a low-order SMO for the average-state estimation, as an application of the Utkin's observer [24], with additional Luenberger-type gains and subjected to an unknown input vector. Notice that, differently with the standard SMO formulations, our design accounts also the presence of an unmatched perturbations, whose effect needs to be minimized, cf. [19], [20] with [17], [18], [24].

Let $\hat{\boldsymbol{z}}=\left[\hat{a}, \hat{\boldsymbol{y}}^{\top}\right]^{\top}$ be vector of stacking the estimation of $\boldsymbol{z}=\left[a, \boldsymbol{y}^{\top}\right]^{\top}$, the proposed "Average-State SMO" is

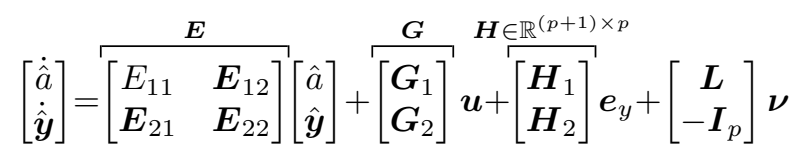

where $\boldsymbol{H}_{i}$ with $i=1,2$, and $\boldsymbol{L}$ are design matrices with proper dimensions, and

$$
e_{a}=\hat{a}-a, \quad \boldsymbol{e}_{y}=\hat{\boldsymbol{y}}-\boldsymbol{y} .
$$

denote the observer's estimation errors and $\nu \in \mathbb{R}^{p}$ is a discontinuous vector defined as

$$
\boldsymbol{\nu}=\rho \operatorname{sign}\left(\boldsymbol{e}_{y}\right), \text { with } \rho>0 .
$$

Theorem 1 Consider an LSS as in Eq. (2), its lower order projection in Eq. (5) and let Assumptions 1-2 be in force. Let the design of the Average-State SMO in Eq. (8) be

$$
\left\{\begin{aligned}
\boldsymbol{H}_{1} & =-\boldsymbol{E}_{12} \\
\boldsymbol{H}_{2} & =\boldsymbol{E}_{22}^{s}-E_{22} \quad \text { with } \operatorname{eig}\left(\boldsymbol{E}_{22}^{s}\right) \subset \mathbb{C}_{<0}^{p} \\
\boldsymbol{L} & =\left(\alpha \mathbf{1}_{k}^{\top}-\boldsymbol{F}_{1}\right) \boldsymbol{F}_{2}^{+} \quad \text { with } \alpha \in \mathbb{R} \\
\rho & >\left\|\boldsymbol{E}_{21} a(t)\right\|_{\infty}+\left\|\boldsymbol{F}_{2} \boldsymbol{\delta}(t)\right\|_{\infty}, \quad \forall t \geq 0
\end{aligned}\right.
$$

If and only there exists $\alpha \in \mathbb{R}$ such that

$$
\begin{aligned}
& \operatorname{rank}\left(\left[\begin{array}{c}
\alpha \mathbf{1}_{k}^{\top}-\boldsymbol{F}_{1} \\
\boldsymbol{F}_{2}
\end{array}\right]\right)=\operatorname{rank}\left(\boldsymbol{F}_{2}\right) \\
& \alpha<\left(\boldsymbol{F}_{1}\left(\boldsymbol{F}_{2}^{+} \boldsymbol{F}_{2}-\boldsymbol{I}_{k}\right) \mathbf{1}_{k}\right) /\left(\mathbf{1}_{k}^{\top} \boldsymbol{F}_{2}^{+} \boldsymbol{F}_{2} \mathbf{1}_{k}\right),
\end{aligned}
$$

then, for any initial condition, the estimation error on the average profile converges exponentially to zero with rate

$$
\beta(\alpha)=\left(\boldsymbol{F}_{1}-\alpha \mathbf{1}_{k}^{\top}\right)\left(\boldsymbol{I}_{k}-\boldsymbol{F}_{2}^{+} \boldsymbol{F}_{2}\right) \mathbf{1}_{k}^{\top}+k \alpha .
$$

Proof: The first part of the proof will shows the design in Eq. (11) may allow the average-state estimation. Then, the second part proves the necessity and sufficiency of Eqs. (12)(13) for the exact asymptotic average-state estimation.
(Part 1) By differentiating Eq. (9), one obtains

$$
\begin{aligned}
& \dot{e}_{a}=E_{11} e_{a}-\boldsymbol{F}_{1} \boldsymbol{\delta}+\boldsymbol{L} \boldsymbol{\nu} \\
& \dot{\boldsymbol{e}}_{y}=\boldsymbol{E}_{21} e_{a}+\boldsymbol{E}_{22}^{s} \boldsymbol{e}_{y}-\boldsymbol{F}_{2} \boldsymbol{\delta}-\boldsymbol{\nu} .
\end{aligned}
$$

Since $\dot{e}_{a}$ is decoupled from $\boldsymbol{e}_{y}$, and the unknown input $\boldsymbol{\delta}(t)$ is bounded by Assumption 2, and $\boldsymbol{E}_{22}^{s}$ can be arbitrarily designed such that $\operatorname{eig}\left(\boldsymbol{E}_{22}^{s}\right) \subset \mathbb{C}_{<0}^{p}$, then $\boldsymbol{e}_{y}$ can be driven to zero, in a finite-time by means of the non-smooth control $\nu$ in Eq. (10). To show this, let us consider the candidate Lyapunov function $V=\frac{1}{2} \boldsymbol{e}_{y}^{\top} \boldsymbol{e}_{y}$. By differentiating it along the trajectories of $\boldsymbol{e}_{y}$, one obtains $\dot{V}=\boldsymbol{e}_{y}^{\top} \dot{\boldsymbol{e}}_{y}<-\varepsilon \sqrt{V}<0$, with $\varepsilon>0$, if and only if $\rho \geq\left\|\boldsymbol{E}_{21} z_{1}\right\|_{\infty}+\left\|\boldsymbol{F}_{2} \bar{\delta}\right\|_{\infty}+\varepsilon$. This implies that an ideal sliding motion on $\dot{\boldsymbol{e}}_{y}=\boldsymbol{e}_{y}=$ $\mathbf{0}_{p}$ is guaranteed to take place in finite-time if Eq. (11) is satisfied. Thus, during the sliding-motion, it further results the average-state error dynamic takes the form

$$
\dot{e}_{a}=E_{11} e_{a}-\boldsymbol{F}_{1} \boldsymbol{\delta}+\boldsymbol{L} \boldsymbol{\nu}_{e q} \quad 0=\boldsymbol{E}_{21} e_{a}-\boldsymbol{F}_{2} \boldsymbol{\delta}-\boldsymbol{\nu}_{e q}
$$

Substituting for $\nu_{e q}$ yields

$$
\dot{e}_{a}=\left(E_{11}+\boldsymbol{L} \boldsymbol{E}_{21}\right) e_{a}-\left(\boldsymbol{F}_{1}+\boldsymbol{L} \boldsymbol{F}_{2}\right) \boldsymbol{\delta} .
$$

By Assumption 1, and by means of the Popov-BelevitchHautus rank test [24], we conclude that $\boldsymbol{E}_{21} \neq 0$. The latter condition confirms the possibility to estimate the averagestate under certain conditions on Eq. (5) and on $\boldsymbol{L}$ in Eq. (11). (Part 2) The average-state estimation error converges exponentially if and only if the next two condition hold true

$$
\begin{aligned}
& \left(\boldsymbol{F}_{1}+\boldsymbol{L} \boldsymbol{F}_{2}\right) \boldsymbol{\delta}(t)=0, \quad \forall t \in \mathbb{R} \\
& E_{11}+\boldsymbol{L} \boldsymbol{E}_{21}<0 .
\end{aligned}
$$

Due to the structure of $\boldsymbol{\delta}(t)$ and its property in Eq. (6), one has that Eq. (18) holds if and only if $\boldsymbol{L} \in \mathbb{R}^{p \times 1}$ is such that

$$
\boldsymbol{F}_{1}+\boldsymbol{L} \boldsymbol{F}_{2}=\alpha \mathbf{1}_{k}^{\top}, \quad \alpha \in \mathbb{R} .
$$

By the Rouché-Capelli theorem, the above linear system is well-posed with respect to vector of parameters in $\boldsymbol{L}$ if and only if the condition in Eq. (12) holds. In such case, the design of $\boldsymbol{L}$ given in Eq. (11) is the solution to the system of equations and the error dynamics reduces to

$$
\dot{e}_{a}=\left(E_{11}+\boldsymbol{L} \boldsymbol{E}_{21}\right) e_{a}=\beta(\alpha) e_{a},
$$

from which the condition in Eq. (13) and the expression of the convergence rate in Eq. (14) can be derived by substitution. Summing up, the error $e_{a}$ can be made asymptotically stable if and only if Eqs. (18)-(19) hold true, which we have proved to be equivalent to Eqs. (12)-(13).

The next result provides some additional requirements on the low-order projection of the LSS enabling the exact average estimation at any arbitrary convergence rate.

Theorem 2 Consider an LSS as in Eq. (2), its lower order projection in Eq. (5) and let Assumptions 1-2 be in force. Let the design of the Average-State SMO in Eq. (8) be the one in Eq. (11), and let condition in Eq. (12) be in force. If and only if the next relation is satisfied

$$
\operatorname{rank}\left(\left[\begin{array}{c}
\mathbf{1}_{k}^{\top} \\
\boldsymbol{F}_{1}
\end{array}\right]\right)=1,
$$


then the convergence rate of the average-state estimation given by Eq. (14) can be arbitrarily chosen through $\alpha$.

Proof: The convergence rate of the average-state error $\beta(\alpha)$ in Eq. (21) results to be a function of $\alpha$. Thus, it could be chosen arbitrarily if and only if $\alpha$ can be chosen arbitrarily as well. Moreover, notice that $\alpha$ can be chosen arbitrarily if and only if condition in Eq. (22) holds true. Indeed, since in this case $\alpha$ does not appear anymore in Eq. (12), that rank condition reduces to

$$
\operatorname{rank}\left(\left[\begin{array}{c}
\boldsymbol{F}_{1} \\
\boldsymbol{F}_{2}
\end{array}\right]\right)=\operatorname{rank}\left(\boldsymbol{F}_{2}\right)
$$

which concludes this proof.

Notice that, in real applications it is likely that an LSS as in Eq. (2) does not satisfy the condition in Eq. (12), thus preventing the achievement of the exact average-state estimation. Such condition becomes even less realistic as the size of the system becomes larger, since it implicitly it requires that every unmeasured node is connected to at least one output node, that goes instead in contrast with the reality where only a limited number of measurement node is available. Thus motivated, in the next theorem, we show that even if Eq. (12) is not fulfilled, the same observer design still provides a good estimation of the average-state by ensuring a bounded steady-state error.

Theorem 3 Consider an LSS as in Eq. (2), its lower order projection in Eq. (5) and let Assumptions 1-2 be in force. Let the design of the Average-State SMO in Eq. (8) be the one in Eq. (11) and assume condition in Eq. (12) does not hold. Then, the estimation error on the average-state converges asymptotically within a boundary layer whose size is $\bar{e}_{a}$, i.e.,

$$
\limsup _{t \rightarrow \infty}\left\|e_{a}(t)\right\| \leq \bar{e}_{a} .
$$

Proof: We recall that the dynamics of the average-state error are governed by Eq. (17),

$$
\dot{e}_{a}=\beta(\alpha) e_{a}-\left(\boldsymbol{F}_{1}+\boldsymbol{L} \boldsymbol{F}_{2}\right) \boldsymbol{\delta}
$$

where $\beta(\alpha)$ is as in Eq. (14). Since condition in Eq. (12) is no more fulfilled, the effect of the unmatched unknown inputs, due to $\boldsymbol{\delta}(t)$, can not be completely rejected. Nevertheless, the average error trajectory $e_{a}(t)$ satisfies

$$
\left|e_{z}(t)\right| \leq\left|e^{\beta(\alpha) t} e_{z}(0)\right|+\left|\int_{0}^{t} e^{\beta(\alpha)(t-\tau)}\left(\boldsymbol{F}_{1}+\boldsymbol{L} \boldsymbol{F}_{2}\right) \boldsymbol{\delta}(\tau) d \tau\right| .
$$

Due to Eq. (13), the term $\beta(\alpha)$ is strictly negative, and thus $\lim _{t \rightarrow \infty}\left|e^{\beta(\alpha) t}\right|=0$. Thus, one derives that

$$
\limsup _{t \rightarrow \infty}\left|e_{a}(t)\right| \leq \limsup _{t \rightarrow \infty} \int_{0}^{t}\left|e^{\beta(\alpha)(t-\tau)}\right|\left|\left(\boldsymbol{F}_{1}+\boldsymbol{L} \boldsymbol{F}_{2}\right) \boldsymbol{\delta}(\tau)\right| d \tau .
$$

Since the two functions argument of the above integral are non-negative by definition, it holds that (see [25, Sect. 23])

$\limsup _{t \rightarrow \infty}\left|e_{a}(t)\right| \leq \max _{\tau \geq 0}\left|\left(\boldsymbol{F}_{1}+\boldsymbol{L} \boldsymbol{F}_{2}\right) \boldsymbol{\delta}(\tau)\right| \cdot \lim _{t \rightarrow \infty} \int_{0}^{t}\left|e^{\beta(\alpha)(t-\tau)}\right| d \tau$.

Finally, by a change of variable and solving the definite integral we can compute

$$
\limsup _{t \rightarrow \infty} \int_{0}^{t}\left|e^{\beta(\alpha)(t-\tau)}\right| d \tau=\int_{0}^{\infty}\left|e^{\beta(\alpha)(\tau)}\right| d \tau=\frac{1}{|\beta(\alpha)|}
$$

Then, by letting $\boldsymbol{\varphi}(\alpha, \boldsymbol{L})=\boldsymbol{F}_{1}+\boldsymbol{L} \boldsymbol{F}_{2}-\alpha \mathbf{1}_{k}^{\top}$, we can further find an upper-bound to the average-state error as

$$
\begin{aligned}
\limsup _{t \rightarrow \infty}\left|e_{a}(t)\right| & \leq \frac{1}{|\beta(\alpha)|} \max _{\tau \geq 0}\left|\left(\boldsymbol{F}_{1}+\boldsymbol{L} \boldsymbol{F}_{2}\right) \boldsymbol{\delta}(\tau)\right| \\
& \leq \frac{1}{|\beta(\alpha)|} \max _{\tau \geq 0}\left|\left(\alpha \mathbf{1}_{k}^{\top}+\boldsymbol{\varphi}(\alpha, \boldsymbol{L})\right) \boldsymbol{\delta}(\tau)\right| \\
& \leq \frac{\|\boldsymbol{\varphi}(\alpha, \boldsymbol{L})\|}{|\beta(\alpha)|} \max _{\tau \geq 0}\|\boldsymbol{\delta}(\tau)\|=\bar{e}_{a}
\end{aligned}
$$

where in the last step we have exploited the property in Eq. (7), and the proof is complete by considering that $\boldsymbol{\delta}(t)$ is bounded due to Assumption 2 .

In the next corollary is proved that the estimation provided by the proposed Average-State SMO is optimal in the sense that it minimizes the effect of the unmatched unknown inputs.

Proposition 1 Consider an LSS as in Eq. (2), its lower order projection in Eq. (5) and let Assumptions 1-2 be in force. Let the design of the Average-State SMO in Eq. (8) be the one in Eq. (11). Then, the bound on the average estimation error $\bar{e}_{a}$ is minimized by the design of $\boldsymbol{L}$ given in Eq. (11).

Proof: We write $\bar{e}_{a}$ in Eq. (24) as a function $\boldsymbol{L}$,

$$
\bar{e}_{a}=\frac{\left\|\boldsymbol{F}_{1}+\boldsymbol{L} \boldsymbol{F}_{2}-\alpha \mathbf{1}_{k}^{\top}\right\|}{|\beta(\alpha)|} \max _{t \geq 0}\|\boldsymbol{\delta}(t)\| .
$$

The bound on the error can be minimized by the choice of $\boldsymbol{L}$ by solving the following least square problem

$$
\boldsymbol{L}^{*}=\underset{\boldsymbol{L} \in \mathbb{R}^{1 \times p}}{\operatorname{argmin}}\left\|\boldsymbol{F}_{1}+\boldsymbol{L} \boldsymbol{F}_{2}-\alpha \mathbf{1}_{k}^{\top}\right\| .
$$

Then, according to the Projection's Theorem [26, p. 51], or similarly with [20, Proposition 2], the optimal $\boldsymbol{L}^{*}$ is $\boldsymbol{L}^{*}=$ $\left(\alpha \mathbf{1}_{k}^{\top}-\boldsymbol{F}_{1}\right) \boldsymbol{F}_{2}^{+}$, which corresponds to that in Eq. (11).

From the constructing proofs and results of the previous theorem and proposition, it is straightforward notice that not only the average-state error is bounded by $\bar{e}_{a}$ in Eq. (24), but that bound depends by $\alpha$, which is a design parameter. Therefore, in the next corollary we discuss how to make $\alpha$ optimal to minimize the steady-state error bound.

Corollary 1 Consider an LSS as in Eq. (2), its lower order projection in Eq. (5) and let Assumptions 1-2 be in force. Let the design of the Averare-State SMO in Eq. (8) be the one in Eq. (11). The bound on the average estimation error is minimized if $\alpha=\alpha^{*}$, where $\alpha^{*}$ is the solution of

$$
\begin{array}{ll}
\underset{\alpha}{\operatorname{argmin}} & \frac{\|\boldsymbol{\varphi}(\alpha)\|}{|\beta(\alpha)|} \\
\text { subj. to } & \beta(\alpha)<0
\end{array}
$$

where $\beta(\alpha)=\frac{\boldsymbol{\varphi}(\alpha)}{\left(\boldsymbol{F}_{1}-\alpha \mathbf{1}_{k}^{\top}\right)\left(\boldsymbol{I}-\boldsymbol{F}_{2}^{+} \boldsymbol{F}_{2}\right)} \mathbf{1}_{k}^{\top}+k \alpha$.

Notice that although $\alpha^{*}$ minimize the steady-state error upper-bound $\bar{e}_{a}$, it influences also $\beta(\alpha)$. Thus, the optimal 
choice $\alpha=\alpha^{*}$ may have adverse effects on the convergence rate of $e_{a}$ in accordance with Eq. (23). Thus, with aim to trade-off those two performance metrics, the following gain adaptation law is proposed

$$
\dot{\alpha}(t)= \begin{cases}\lambda & \text { if }\left\|\boldsymbol{e}_{y}(t)\right\|<\epsilon \text { and } \alpha(t) \geq \alpha^{*} \\ 0 & \text { otherwise }\end{cases}
$$

where $\lambda<0$, and $0<\epsilon<\left\|\boldsymbol{e}_{y}(0)\right\|$, and $\alpha^{*}<\alpha(0)$, such that Eq. (13) holds true. Intuitively, Eq. (27) adapts $\alpha$ dynamically in order to guarantee, a faster convergence rate equal to $\beta(\alpha(0))$ during the transient regime, while preserving the same optimal steady-state accuracy. In fact, as soon as $\left\|e_{y}(t)\right\|<\epsilon$ is verified, $\alpha$ starts to decrease with rate $\lambda$ until $\alpha^{*}$ is reached. Let us further note that, in accordance with the Proof of Theorem $1,\left\|\boldsymbol{e}_{y}(t)\right\| \equiv 2 V(t)$ is a monotonically decreasing function.

Nonetheless, notice that although $\nu$ in Eq. (10) ideally switches with infinite frequency, in practice due its digital implementation only a finite switching frequency can be achieved. This leads to a small numerical chattering on $\boldsymbol{e}_{y}(t)$, whose size is of the same order of the SMO sampling time $T_{s}$. Thus, particular attention should be paid in choosing $\epsilon$ at least one order bigger than $T_{s}$, to avoid a too slow convergence of $\alpha(t)$ to $\alpha^{*}$. Alternately, and the price to reduce the observer robustness, the mentioned chattering could be completely removed by replacing the sign-operator in Eq. (10) by means of its sigmoid approximation [24].

\section{NUMERICAL SIMULATIONS}

The effectiveness of the proposed Average-State SMO is confirmed through simulation on two test cases, a compartmental system and a reaction-diffusion process. Simulations were performed on the MATLAB ${ }^{\circledR} /$ SIMULINK environment, with the Euler fixed-step solver and $T_{s}=10^{-4}$ seconds.

Example 1 Consider the compartmental system of $n=11$ compartments in [27], whose interconnections are as in Fig. 1a, and whose associated Laplacian Matrix is $\mathcal{L} \in \mathbb{R}^{n \times n}$. Let $x_{i}(t)$ be the state of compartment $i$, and assumed only the grey nodes are available for measurements. This leads the system in the standard form in Eq. (2) where $\boldsymbol{A}=-\mathcal{L}, \boldsymbol{B}=\boldsymbol{C}^{\boldsymbol{\top}}=\left[\mathbf{0}_{3 \times 8}, \boldsymbol{I}_{3}\right]$, $x_{i}(0) \in[0,3]$ and $i=1,2, \ldots, 11$, whereas the LSS's

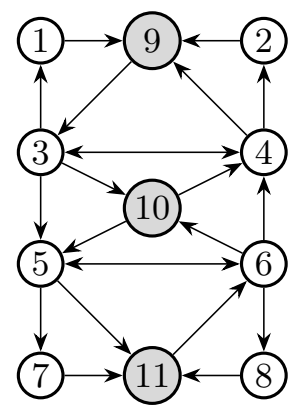

(a) Compartmental system

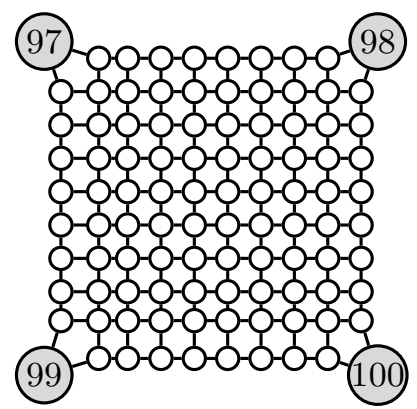

(b) Reaction-diffusion process
Fig. 1: Topologies of the LSSs considered in the simulations.

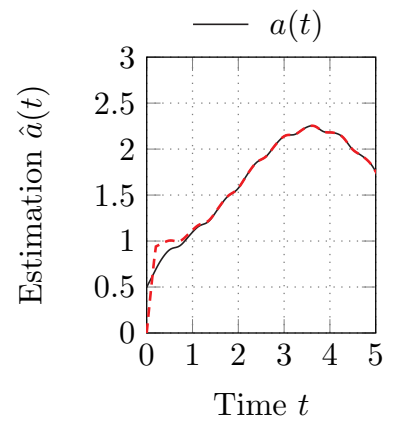

(a)

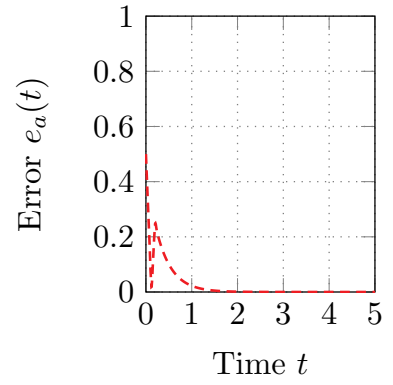

(b)
Fig. 2: Example 1: (a) True average-state profile (solid line) and its estimation (dashed line); (b) Average estimation error.

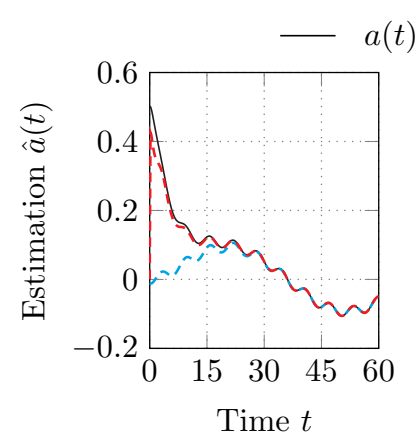

(a)

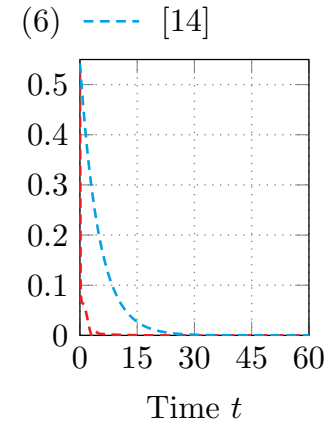

(b)
Fig. 3: Example 2: (a) Average state (solid line) and their estimation (dashed lines); (b) Average-state error. Red dashed lines correspond to the AverageState SMO, while the blue dashed lines to observer proposed in [14].

inputs are $u_{\ell}(t)=10 \sin ((\ell-1) 10 t)$ and $\ell=1,2,3$. By partitioning $\boldsymbol{A}$ as in Eq. (2), it can be noticed that the necessary and sufficient condition in Eq. (12) of Theorem 1 holds, since $\mathbf{1}_{8}^{\top} A_{11}=\mathbf{1}_{8}^{\top} A_{21}=\mathbf{1}_{8}^{\top}$, thus enabling the exact average estimation. In accordance with Theorem 1, the following valid tuning for the proposed SMO is derived, namely, $\boldsymbol{L}^{\top}=-0.25 \mathbf{1}_{8}^{\top}, \rho=5, \boldsymbol{E}_{22}^{s}=-10 \boldsymbol{I}_{3}$, whereas $\alpha-0.375$ is chosen in accordance with Eq. (13), and such that an arbitrarily convergence rate $\beta=-3$ was achieved.

The results of the simulation are shown in Fig. 2. In particular, in Fig. 2a is shown the temporal profile of the true average-state $a(t)$ and that of its estimation $\hat{a}(t)$, through the proposed SMO. The profile of the resulting average-state error is instead depicted in Fig. 2b. As expected, after a finitetime $\boldsymbol{e}_{y}$ is element-wise zero, then the average estimation error approaches zero with the desired exponential rate.

Example 2 Consider the reaction-diffusion process studied in [28] and accounting $n=100$ substances. The diffusion of substances is modelled as undirected graph whose topology is shown in Fig. $1 \mathrm{~b}$, and where the state of the $i$-th node is $x_{i}(t)$. As for Example 1, only the states of the grey nodes are assumed to be available for measurement, thus leading the system in the standard form in Eq. (2) with $\boldsymbol{A}=-\boldsymbol{Q \mathcal { L }}-\boldsymbol{R}, \boldsymbol{C}=\left[\mathbf{0}_{96 \times 4}, \boldsymbol{I}_{4}\right], x_{i}(0) \in[0,3]$, with $i=1,2, \ldots, 100$, whereas $\boldsymbol{R}=0.2 \boldsymbol{I}_{100}$, and $\boldsymbol{Q}=\boldsymbol{I}_{100}$, account for the diffusion, and reaction phenomenon. Finally, 
the system's inputs are selected, time-varying, as follows

$$
\begin{array}{ll}
u_{1}(t)=\sin (0.1 t) & \text { applied to nodes } 97 \text { and } 98, \\
u_{2}(t)=\sin (t) & \text { applied to nodes } 99 \text { and } 100, \\
u_{3}(t)=1 & \text { applied to the other boundary nodes. }
\end{array}
$$

By partitioning $\boldsymbol{A}$ as in Eq. (2), results that $\boldsymbol{A}_{11}$ and $\boldsymbol{A}_{21}$ do not satisfy Eq. (12) of Theorem 1, thus preventing the exact estimation task. We thus consider the proposed gain adaptation law in Eq. (27), with $\lambda=-0.1, \epsilon=0.01$ and $\alpha(0)=-0.005$ which guarantees an higher convergence rate at the transient-regime than that with $\alpha^{*}=-2.1 \times 10^{-3}$, that is $\beta(\alpha(0)) \approx-2.7$. Then, $\alpha(t)$ will asymptotically approach $\alpha^{*}$ thus minimizing the average-state steady-state error upper-bound. Finally, the remaining observer parameters are were $\rho=5$ and $\boldsymbol{E}_{22}^{s}=-10 \boldsymbol{I}_{4}$. In Fig. 3a, and Fig. 3b, for comparison's sake, the average-state estimation $\hat{a}(t)$ and the resulting error profile $e_{a}(t)$ obtained by means of the proposed Average-State SMO are finally compared with those obtained by means of the linear observer proposed in [14, Proposition IV.3], which has been also optimally tuned in accordance with [14, Proposition V.2]. It is evident how the parameter $\alpha$ trade-off the estimation convergence time and the expected error upper-bound, as discussed at the end of Section III. Moreover, thanks to the proposed gain adaptation law in Eq. (26) the estimation convergence rate has been strongly speedup while maintaining the optimal its steady-state accuracy.

\section{CONCLUSions}

This letter solves the average-state estimation problem on an LSS with many unmeasurable nodes as the design of a reduced-order SMO with matched and unmatched perturbations. Conditions on the LSS to guarantee the exact estimation are derived. Then, in the case these were not met, the error boundedness is proved. Then, a gain adaptation strategy to optimally minimize the steady-state error upperbound while trading-off its convergence rate is also devised. It is also worth mentioning the complexity of the proposed observer is independent to the number of unavailable nodes, thus keeping the design scalable and computationally treatable even if the system size is large. Decentralized versions of the proposed design are currently under study. Moreover, extensions of the proposed strategy towards LSSs consisting of nonlinear, and possibly high-order nodes as in [29] also appear promising and worthy of further investigation.

\section{REFERENCES}

[1] Y.-Y. Liu, J.-J. Slotine, and A.-L. Barabási, "Controllability of complex networks," Nature, vol. 473, no. 7346, pp. 167-173, 2011.

[2] A. Pilloni, A. Pisano, E. Usai, P. P. Menon, and C. Edwards, "Decentralized state estimation in connected systems," IFAC Proceedings Volumes, vol. 46, no. 2, pp. 421-426, 2013.

[3] A. C. Antoulas, Approximation of large-scale dynamical systems. Philadelphia, PA, USA: SIAM, 2005.

[4] M. U. B. Niazi, C. Canudas-de Wit, and A. Y. Kibangou, "Average state estimation in large-scale clustered network systems," IEEE Control Netw. Syst., vol. 7, no. 4, pp. 1736-1745, 2020.

[5] S. Coogan and M. Arcak, "A compartmental model for traffic networks and its dynamical behavior," IEEE Trans. Autom. Control, vol. 60, no. 10 , pp. 2698-2703, 2015.
[6] N. Martin, P. Frasca, and C. Canudas-de Wit, "Subgraph detection for average detectability of lti systems," IEEE Trans. Netw. Sci. Eng., vol. 7, no. 4, pp. 2787-2798, 2020.

[7] Z. Li, M. Li, and W. Ji, "Modelling the public opinion transmission on social networks under opinion leaders," IOP Conference Series: Earth and Environmental Science, vol. 69, no. 1, p. 012125, 2017.

[8] K. Deng, P. Barooah, P. G. Mehta, and S. P. Meyn, "Building thermal model reduction via aggregation of states," in IEEE American Control Conference (ACC), 2010, pp. 5118-5123.

[9] T. Sadamoto, T. Ishizaki, and J.-i. Imura, "Average state observers for large-scale network systems," IEEE Control Netw. Syst., vol. 4, no. 4, pp. 761-769, 2017.

[10] _ "Low-dimensional functional observer design for linear systems via observer reduction approach," IEEE Conference on Decision and Control, pp. 776-781, 2013.

[11] P. Murdoch, "Observer design for a linear functional of the state vector," IEEE Trans. Autom. Control, vol. 18, no. 3, pp. 308-310, 1973.

[12] T. L. Fernando, H. M. Trinh, and L. Jennings, "Functional observability and the design of minimum order linear functional observers," IEEE Trans. Autom. Control, vol. 55, no. 5, pp. 1268-1273, 2010.

[13] M. U. B. Niazi, C. Canudas-de Wit, and A. Y. Kibangou, "Average observability of large-scale network systems," IEEE European Control Conference (ECC), pp. 1506-1511, 2019.

[14] M. U. B. Niazi, D. Deplano, C. Canudas-de Wit, and A. Y. Kibangou, "Scale-free estimation of the average state in large-scale systems," IEEE Contr. Syst. Lett., vol. 4, no. 1, pp. 211-216, 2019.

[15] U. Pratap, C. Canudas-de Wit, and F. Garin, "Outlier detection and trimmed-average estimation in network systems," European Journal of Control, 2021.

[16] M. U. B. Niazi, C. Canudas-de Wit, and A. Y. Kibangou, "State variance estimation in large-scale network systems," in IEEE Conference on Decision and Control (CDC). IEEE, 2020, pp. 6052-6057.

[17] S. K. Spurgeon, "Sliding mode observers: a survey," Int. Journal of Systems Science, vol. 39, no. 8, pp. 751-764, 2008.

[18] T. Floquet, C. Edwards, and S. K. Spurgeon, "On sliding mode observers for systems with unknown inputs," Int. Journal of Adapt. Control Signal Process., vol. 21, no. 8-9, pp. 638-656, 2007.

[19] A. F. De Loza, F. J. Bejarano, and L. Fridman, "Unmatched uncertainties compensation based on high-order sliding mode observation," Int. Journal of Robust Nonlinear Control, vol. 23, no. 7, pp. 754-764, 2013.

[20] F. Castaños and L. Fridman, "Analysis and design of integral sliding manifolds for systems with unmatched perturbations," IEEE Trans. Autom. Control, vol. 51, no. 5, pp. 853-858, 2006.

[21] T. Ishizaki, K. Kashima, J.-i. Imura, and K. Aihara, "Model reduction and clusterization of large-scale bidirectional networks," IEEE Trans. Autom. Control, vol. 59, no. 1, pp. 48-63, 2014.

[22] G. Di Muro, F. G. Murphy, R. D. Vann, and L. E. Howle, "Are interconnected compartmental models more effective at predicting decompression sickness risk?" Inform. Med. Unlocked, vol. 20, no. 100334, 2020.

[23] A. Pilloni, A. Pisano, Y. Orlov, and E. Usai, "Consensus-based control for a network of diffusion pdes with boundary local interaction," IEEE Trans. Autom. Control, vol. 61, no. 9, pp. 2708-2713, 2015.

[24] C. Edwards and S. Spurgeon, Sliding mode control: Theory and applications. Crc Press, 1998.

[25] R. G. Bartle, The elements of real analysis. Wiley: New York, 1964.

[26] D. G. Luenberger, Optimization by vector space methods. John Wiley \& Sons, 1997.

[27] G. G. Walter and M. Contreras, Compartmental modeling with networks. Springer Science \& Business Media, 2012.

[28] M. Arcak, "Certifying spatially uniform behavior in reaction-diffusion pde and compartmental ode systems," Automatica, vol. 47, no. 6, pp. 1219-1229, 2011.

[29] B. Wang, W. Chen, B. Zhang, and Y. Zhao, "Regulation cooperative control for heterogeneous uncertain chaotic systems with time delay: A synchronization errors estimation framework," Automatica, vol. 108, no. 108486, 2019. 